\title{
Animation of Chinese Characters: Evolution of Shapes and Styles*
}

\author{
WANG Ning \\ Beihang University, Beijing, China
}

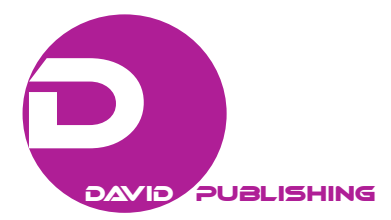

\author{
HU Jia-jia \\ Beijing Normal University, Beijing, China
}

\begin{abstract}
Hanzi (Chinese characters) has a long history and affluent contents. To promote the popularity of historical and cultural knowledge of Chinese characters, an online program has been launched by Beihang University and Beijing Normal University to explain Chinese characters' original meanings and evolution processes with vivid and expressive animation videos. Currently, More than 1,000 videos can be accessed through the website "http://www.chinesecharacter.org/”. Besides these online resources, a human-computer interaction system is also proposed to simulate clerical changes of Chinese characters through computer morphing technology. What we want is to make the teaching and learning of Chinese characters more reasonable, more easily understandable and more interesting.
\end{abstract}

Keywords: animation, evolution of Chinese characters, original meaning, clerical change

\section{Introduction}

Since 2013, a multidisciplinary research project named "Digital Technologies and Systems to Visualize Evolution of Chinese Characters" has been jointly developed by "State Key Laboratory of Virtual Reality Technology and Systems" at Beihang University and "Research Institute of Chinese Characters and Chinese Information Processing” at Beijing Normal University. This paper is one fruit of the big project. The first section gives a brief background. The second section introduces an online program to explain Chinese characters' original meanings and evolution using computer animation technology. The third section proposes a teaching system to simulate clerical changes of Chinese characters through computer morphing technology. And the final section makes a prospect to the research in future.

\section{Background}

Hanzi (Chinese writing) is well known as the representative of ideographic system. That means each Chinese character was formed according to a word's meaning, which is called the character's original meaning. Hanzi is also known as the oldest writing system still in daily use. The earliest confirmed evidence of the Chinese script yet discovered is the body of inscriptions on oracle bones from the Shang Dynasty (1600 B.C.-1100 B.C.). During the long course of development, the shapes and styles of Chinese characters had

\footnotetext{
* Acknowledgements: This paper is supported by National Key Technology Research \& Development Program of China (No.2014BAK18B01) and the Fundamental Research Funds for the Central Universities.

WANG Ning, Associate Professor, School of New Media Art and Design, Beihang University.

HU Jia-jia (Corresponding Author), Lecturer, School of Chinese Language and Literature and Research Center for Folklore, Classics and Chinese Characters, Beijing Normal University.
} 
undergone great changes, which can be divided into five main stages: oracle bone inscription, bronze inscription, small seal script, clerical script and regular script. Figure 1 shows these five stages in the evolution process of Chinese character “友”, which means “friendship” in English.

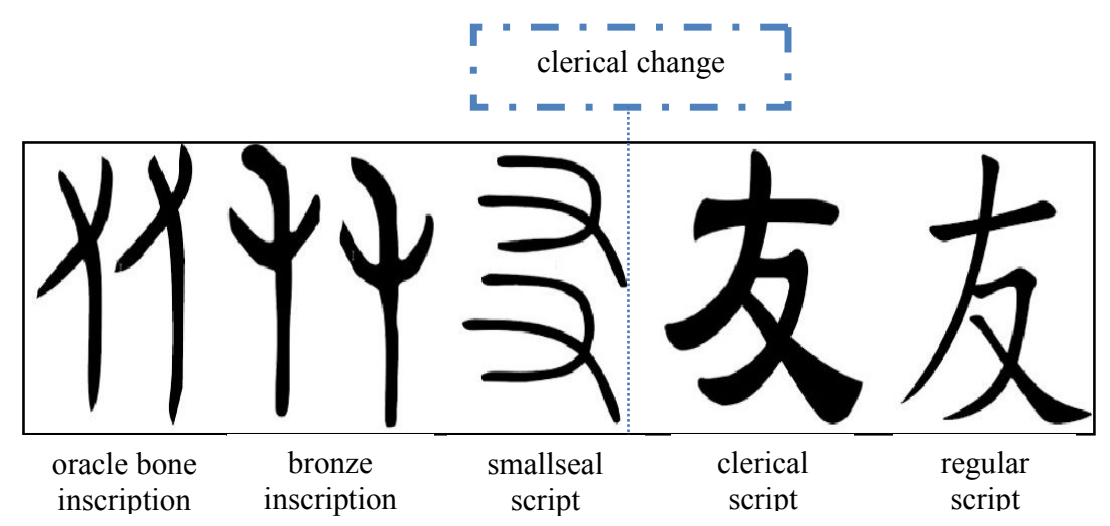

Figure 1. The evolution process of “友”.

The greatest variation, as seen in Figure 1, occurred between small seal script and clerical script, which is called clerical change. Those scripts before the clerical change are classified into ancient scripts, while those ones after the clerical change are classified into modern scripts. The clerical change is so significant that people today, without tutoring, can hardly recognize ancient scripts or understand why a Chinese character was formed like that.

Usually, a traditional method to teach a Chinese character original meaning and its evolution process is to list this character's typical shapes in different stages as the character“友” shown in Figure 1. The teacher would tell students that “狅” is the oracle bone inscription of “友”. It is composed of two “ $\nmid$ ” which looks like as well as means "right hand". Therefore, “友” means "friendship" clearly by means of "hand in hand". However, there are still two questions for the teacher to answer: first, the similarity between " $\ngtr$ "and "right hand"; and second, how the seal script" 곡" becomes the clerical script “友”. Sometimes, a still image can be used to answer the first question. Figure 2 is an illustration (LI, 1992) of the similarity between to " $\nmid$ " and the side outline of a human's right hand.

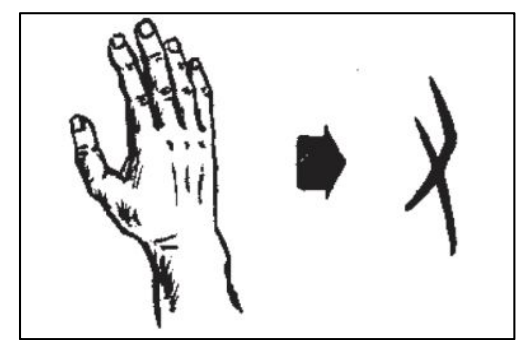

Figure 2. The similarity between "right hand" and " \’”.

The second question would be more difficult to answer, because the change process is dynamic. An alternative way for the teacher is to write down different scripts of “友” on the blackboard with colored chalk to show the correspondence between strokes by means of the same color (or number) as Figure 3 shows. Luckily, it is today, thanks to modern multimedia and virtual technology, possible to show the process vividly. 


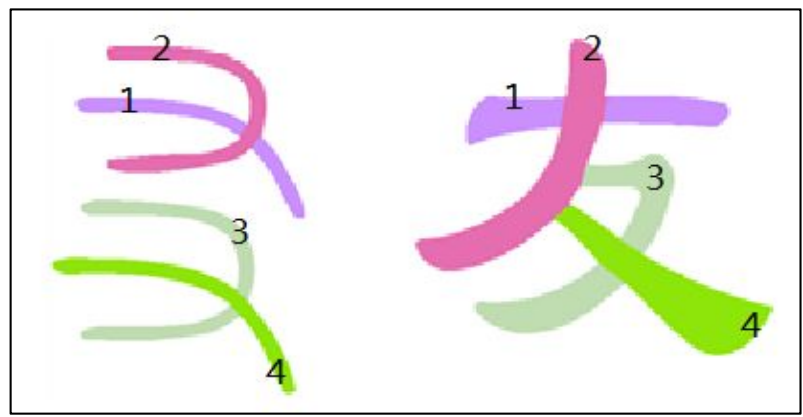

Figure 3. The correspondent strokes of “友” between its small seal script and clerical script.

\section{Animation of Chinese Character Evolution}

Since 2013, a multidisciplinary research project named "Digital Technologies and Systems to Visualize Evolution of Chinese Characters" has been jointly developed by "State Key Laboratory of Virtual Reality Technology and Systems" at Beihang University and "Research Institute of Chinese Characters and Chinese Information Processing" at Beijing Normal University. This project has three primary tasks: (1) to design and construct a marked system of Chinese character information resources; (2) to develop digital technologies and tools for the construction of the above system; (3) to design and develop kinds of software to teach Chinese characters based on the above two works.

"Animation of Chinese Character Evolution" is one application of this big project. It has an online program, ${ }^{1}$ as shown in Figure 4, aimed to explain Chinese characters' original meanings and evolution processes vividly by using computer animation technology. The online program so far has more than 1,000 animation videos of Chinese characters and the number is continuously increasing.

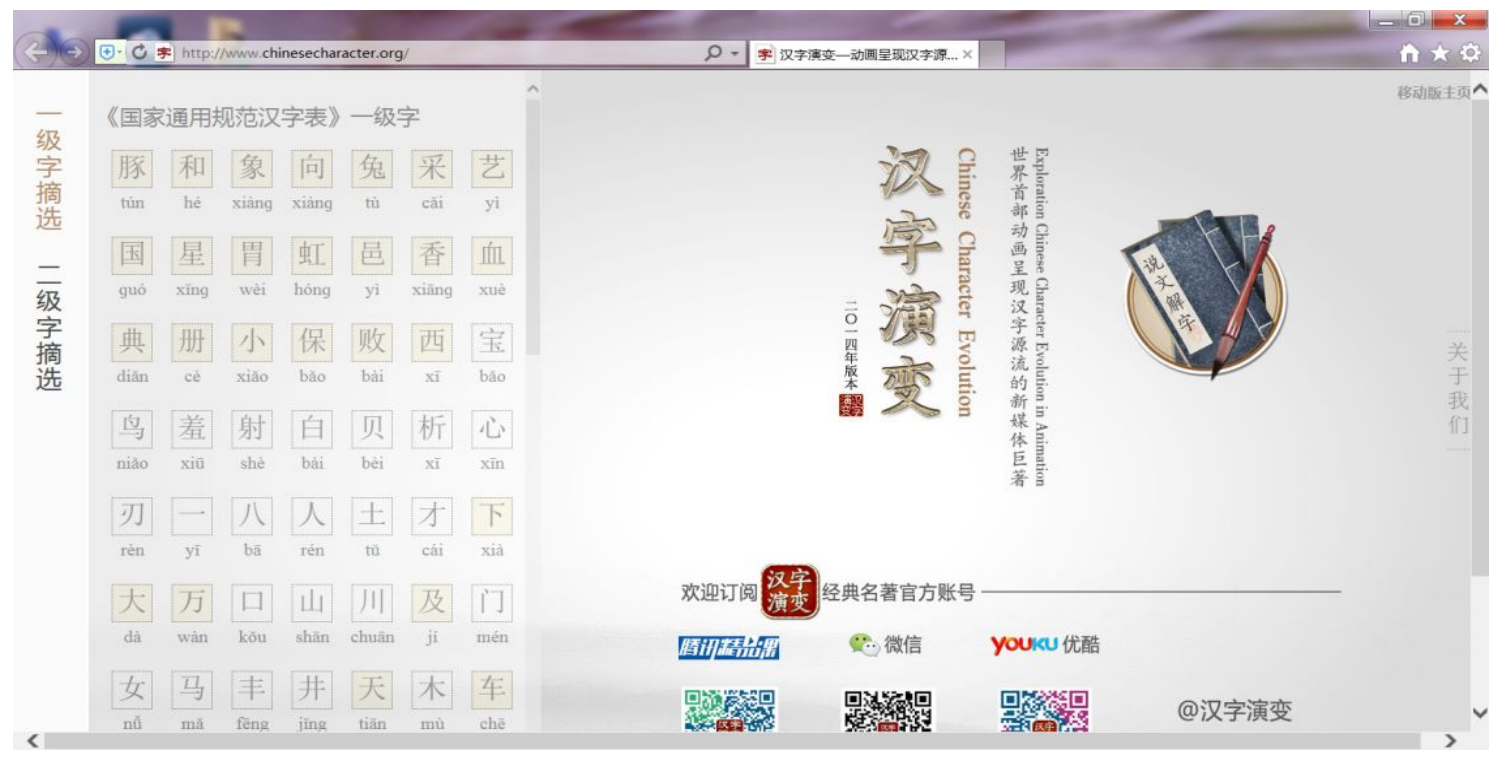

Figure 4. The home page of online "Chinese Character Evolution" program.

Figure 5 is a screenshot of the animated evolution process of Chinese character "马" which means "horse" in English. The evolution process is divided into five main stages: oracle bone inscription, bronze inscription,

\footnotetext{
${ }^{1}$ See from http://www.chinesecharacter.org/.
} 
small seal script, clerical script and regular script. And simplified Chinese characters, like “马”, have six stages.

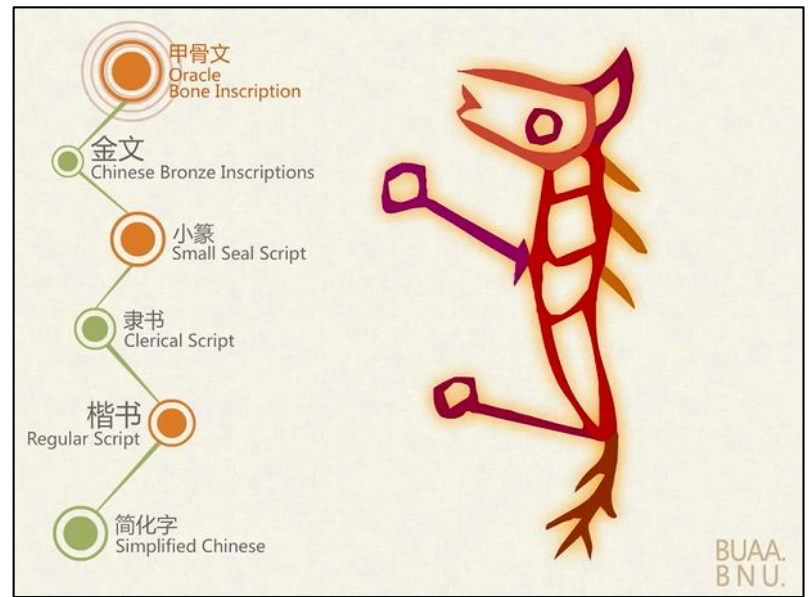

Figure 5. A screenshot of the animated evolution process of “马”.

Each animation video started with an illustration of the character's original meaning. The video of “马”, for example, starts with the image of a horse with soaring spirit. It makes audiences get to know that the oracle bone inscription " o " was actually formed according to the outline of a war horse and how the oracle bone inscription " a continuous and dynamic, it's only possible to present some screenshots of critical points on paper.

Table 1

Screenshots of Critical Points in the Video of “马” to Show Its Animated Evolution Process

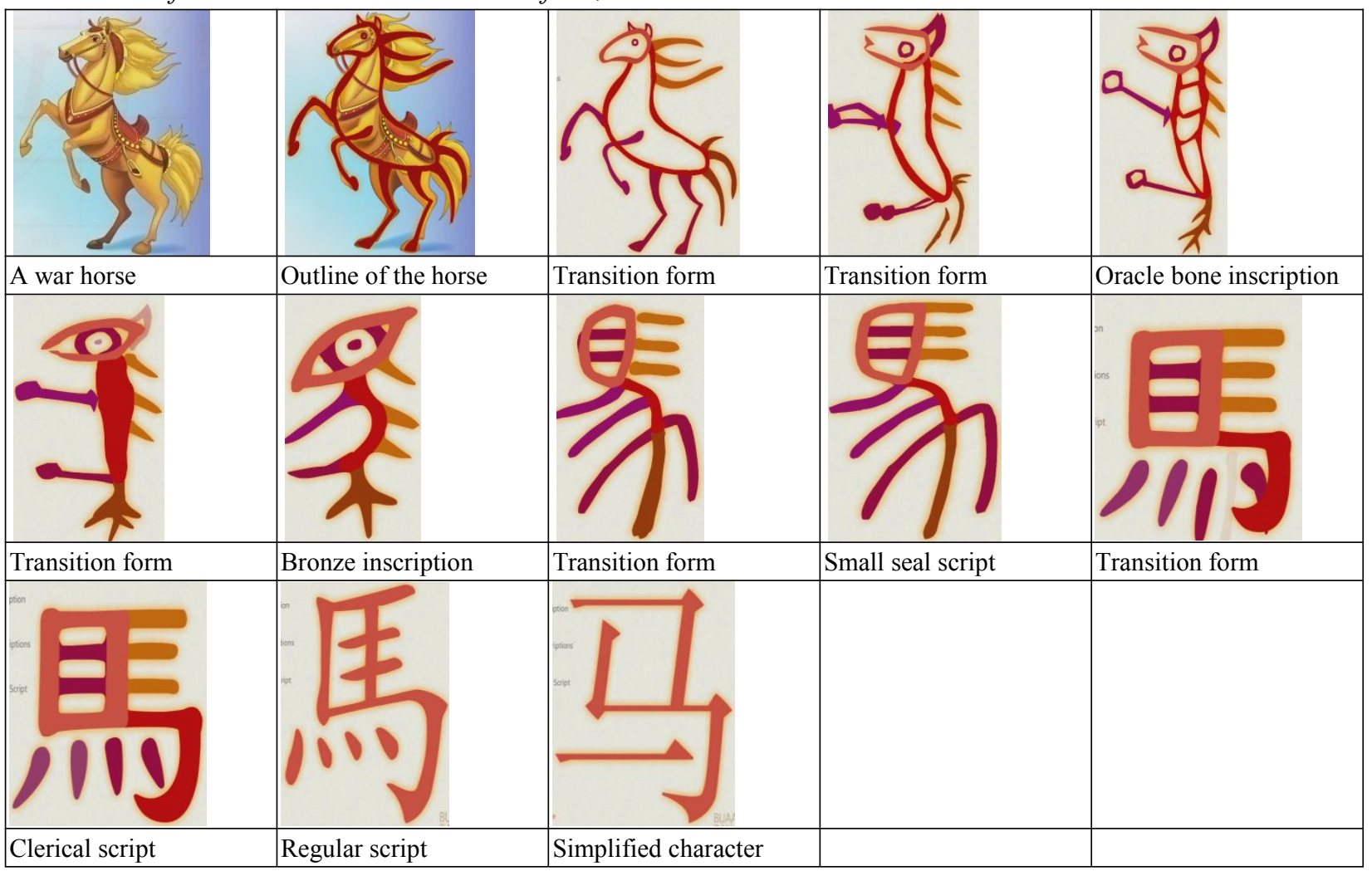




\section{A Morphing System to Simulate Clerical Change}

The animation video can give audiences a direct and interesting impression of each Chinese character's original meaning and evolution process. However, clerical change, the variation between seal script and clerical script, is complicated as well as critical. It is the bridge to communicate ancient scripts and modern scripts, while, it can hardly be mastered only through browsing videos. Therefore, a morphing system is proposed to simulate clerical change for teaching and studying (YUE, LIANG, HU, \& GUO, 2012).

Based on "Structure Theory of Chinese Characters", Chinese characters are made up of components and components are constituted by strokes, as shown in Figure 6. As a result, the clerical change of a Chinese character can be analyzed by the change of strokes.

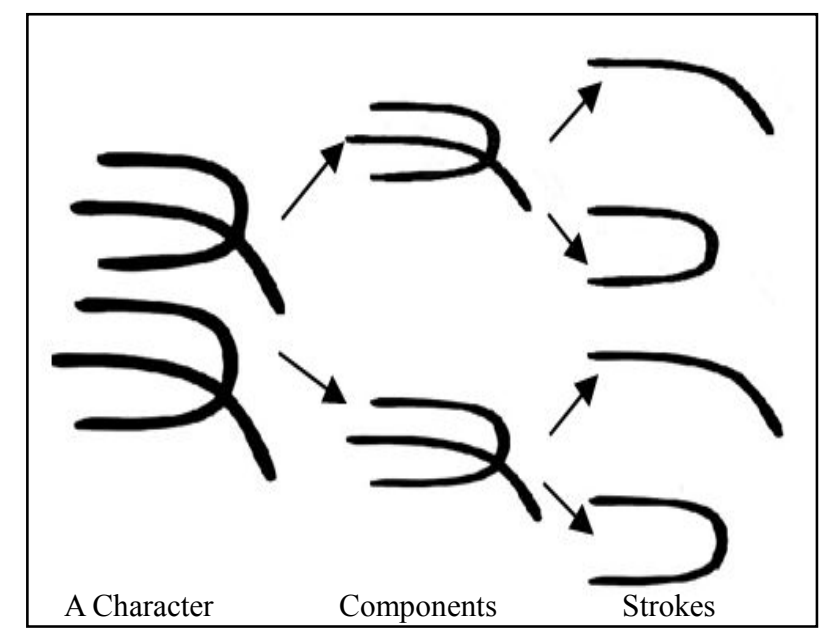

Figure 6. The structure of small seal script “友”.

The morphing system provides an interface for users to define the corresponding relationship of strokes between small seal script and clerical script of a same character. As shown in Figure 7, when the user inputs a Chinese character, the system will present both of its small seal script and clerical script forms, and then extract their strokes. The user can use the toolbar to mark strokes with different colors, and the two strokes with the same color of both scripts are corresponding to each other.

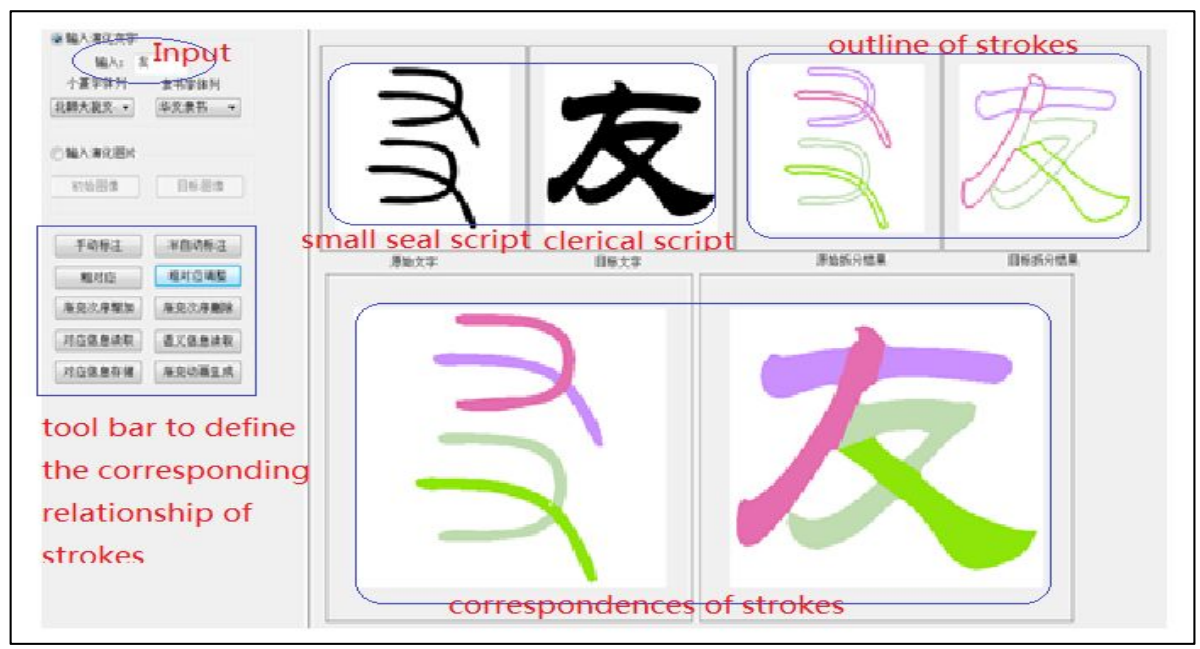

Figure 7. The user interface of the teaching system. 
Based on the correspondence between strokes of different scripts, a morphing process can be generated automatically by computer algorithms, as seen in Figure 8 .

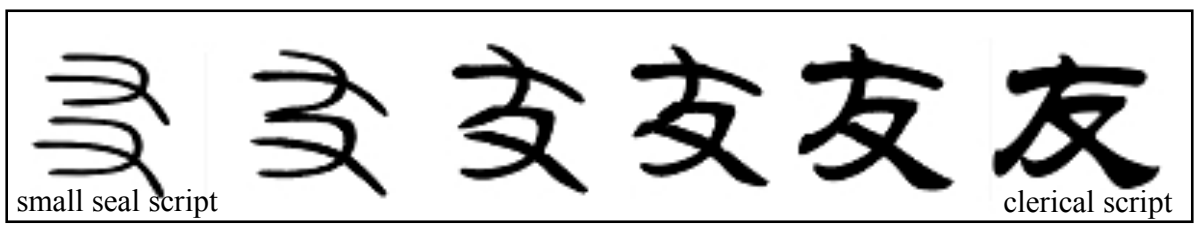

Figure 8. The morphing process of clerical change of “友”.

The corresponding relationships can be saved for further use. Therefore, teachers can use the morphing system to illustrate typical clerical changes on classes again and again. And students can use it to practice on more Chinese characters which can then be examined and corrected by teachers.

\section{Future: Learning Characters in Groups}

Hanzi is a system. The elements of the system are not characters but character components which bring certain meanings when they are used to compose characters. A character composed of only one component, i.e., the character itself, is called non-composite character, like “木” which means "tree” in English. A character composed of two or more components is called composite character, like “林” which means “forest” in English. It is natural to use the non-composite character to understand those composite characters. Our future work is to organize animation videos of separate Chinese characters into networks with selected non-composite characters as cores, as Figure 9 shows, which can reflect the principles of Chinese characters' structures.

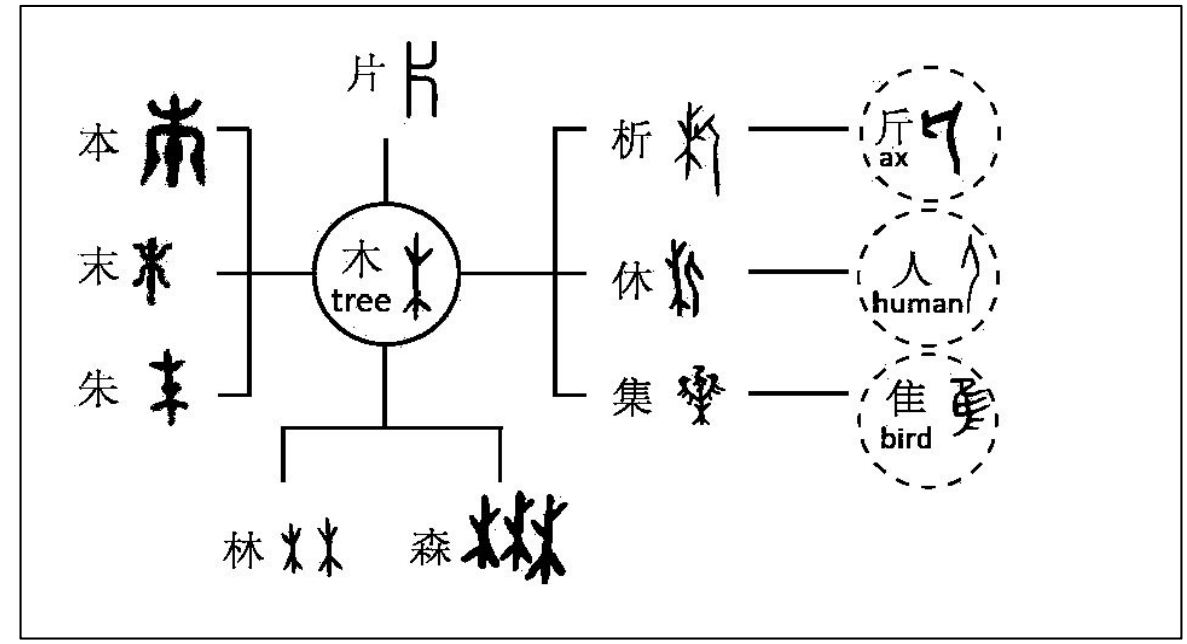

Figure 9. Part of the group of Chinese characters composed by the core character “木”.

\section{Conclusion}

As a multidisciplinary project, "Digital Technologies and Systems to Visualize Evolution of Chinese Characters" requires the usage of various computer technologies and abundant knowledge of Chinese characters and culture. What is the most important is how to select the appropriate technology according to different kinds of Chinese characters' knowledge to make the learning more expressive and interesting. This is a long-term integration process which needs scholars of different academic backgrounds trying hard to understand each other on the basis of their own knowledge structure. 


\section{References}

LI, L. (1992). Tracing the roots of Chinese characters: 500 cases. Beijing: Beijing Languages College Press.

YUE, P., LIANG, X., HU, J., \& GUO, Ch. (2012). Knowledge-based 2D morphing system for Chinese characters of different styles (Paper presented at 25th Annual Conference on Computer Animation and Social Agents, Singapore).

YU, B., LIANG, X., HU, J., \& SUN, L. (2012). Statistical structure modeling and optimal combined strategy based Chinese components recognition (Paper presented at 8th International Conference on Signal Image Technology and Internet Based Systems, Italy).

WANG, X., LIANG, X., HU, J., \& SUN, L. (2012). Stroke-based Chinese character completion (Paper presented at 8th International Conference on Signal Image Technology and Internet Based Systems, Italy).

SUN, L., LIU, M., HU, J., \& LIANG, X. (2014). A Chinese character teaching system using structure theory and morphing technology. PLOS ONE, 9(6), e100987. 